\title{
Generic Computation Method of Free-Molecular Flow Effects on Space Objects
}

\author{
By Takahiro KATO ${ }^{1)}$, Benny RIEVERs ${ }^{1)}$, and Meike LIST ${ }^{1)}$ \\ ${ }^{1)}$ Center of Applied Space Technology and Microgravity (ZARM), University of Bremen, Bremen, Germany
}

(Received July 28th, 2015)

\begin{abstract}
This paper presents an analytical formulation and a computational method of free-molecular flow effects incorporating the temperature variation over the orbital motion of a satellite. The rarefied aerodynamics interactions, so-called free-molecular flow, has been studied for decades and the complexity of the phenomena has been the bottleneck. A new aspect of analysis on the surface temperature contributions is implemented in the analytical form. It affects the magnitude of momentum exchanges and thus the resulting force at the satellite surfaces. The free-molecular interaction perturbs the orbit and the attitude of a satellite as well as of any other orbiting space objects below the exosphere of the Earth, including International Space Station and space debris. The enhanced fidelity and accuracy of the atmospheric perturbation model is expected to complement with the growing requirements on the orbit determination accuracy of space objects, especially gravimetry missions. Under exemplary orbit and attitude conditions, temperature variations together with the free-molecular flow effects are obtained and discussed. The result indicates the magnitude of the force coefficient jump is around $6 \%$, which is induced by the surface temperature variation.
\end{abstract}

Key Words: Free-Molecular Flow, Perturbation Modeling, Simulation, Space Objects

\section{Nomenclature}

$\begin{array}{cll}\alpha & : \text { absorptivity } \\ \gamma & : \text { reflectivity } \\ \rho & : \text { air density } \\ \sigma & : \text { reflection coefficient } \\ A & : \text { surface area } \\ \mathbf{F} & : \text { force vector } \\ k & : \text { Boltzmann constant } \\ m & : \text { molar mass of atmosphere } \\ \mathbf{n} & : \text { unit surface normal vector (inward) } \\ S & : \text { speed ratio } \\ \mathbf{t} & : \text { unit tangential vector from } \mathbf{n} \\ T & : \text { temperature } \\ v & : \text { velocity } \\ \text { Subscripts } & & \\ d & : \text { diffuse } \\ i & : \text { initial } \\ m & : \text { molecular } \\ n & : \text { along } \mathbf{n} \\ s & : \text { specular } \\ t & : \text { along } \mathbf{t} \\ w & : \text { wall }\end{array}$

\section{Introduction}

For space objects which are in Low-Earth orbit (LEO), the non-uniform mass distribution of the Earth dominates the perturbations on orbit and attitude. Non-gravitational forces are mostly caused by momentum exchanges at the satellite surfaces. The most prominent of these forces originate from the interaction of the surface with the photons from the Sun, known as solar radiation pressure (SRP). In addition, molecules and ions of rarefied atmosphere in the thermo / exosphere have similar momentum exchanges with the surface element, so-called free-molecular flow (FMF). In contrast with gravitational perturbations, the aerodynamic and radiation pressure effects are difficult to model since they are not directly measurable, and require several decent knowledge of phenomena, geometry, surface property, and irradiating flux.

This paper investigates the FMF effects on a satellite with on-orbit temperature variation in a near circular orbit of LEO. The FMF perturbs orbit and attitude of satellites as well as of any other orbiting space objects below the exosphere of the Earth, including International Space Station and space debris. The enhanced fidelity and accuracy of the atmospheric perturbation model is expected to complement with the growing requirements on the orbit determination accuracy of these space objects including satellites for gravimetry.

For the sake of accurate orbit propagation, precise gravitational and non-gravitational force models are prerequisite. Several gravimetry missions, e.g. CHAMP (DLR, 2000), 1) GRACE (NASA, 2002, see Fig. 1), ${ }^{2)}$ GOCE (ESA, $2009),{ }^{3)}$ have contributed significantly to understanding the gravitational field of the Earth. Analysis of the highly accurate data provided from these missions is still in progress. However, next generation gravimetry missions are foreseen to achieve even higher, unprecedented accuracy and resolution (both in time and space).

This evolving measurement accuracy is equivalent to strict accuracy requirements on orbit and attitude to extract the scientific outcome. For the sake of optimized outcome from those current and future gravimetry missions, a High Performance Satellite simulator for Satellite Gravimetry (HPS-SG) is under development in the project "geo- $Q$ ".

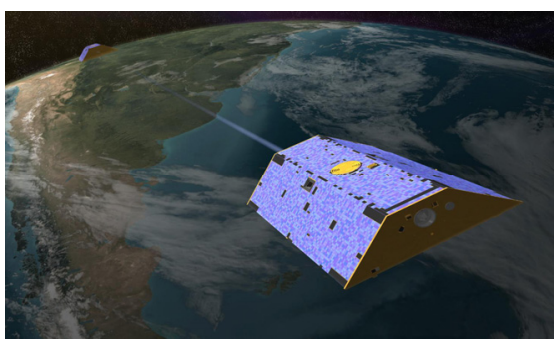

Fig. 1. GRACE satellite pair (Credit : NASA). 
A generic perturbation calculation method has been implemented based on the existing non-gravitational force calculation software developed by Kato et al., ${ }^{4,5)}$ together with the high performance satellite dynamics simulator (HPS) developed at the Center of Applied Space Technology and Microgravity (ZARM). ${ }^{6}$ ) The project geo- $Q$ aims at taking initiative of the high precision and high fidelity mission analysis software development within its scope.

The FMF has been studied for decades and the complexity of the phenomena has been the bottleneck. For example, atmospheric density, ambient temperature, orbital velocity, thermo-optical properties of the object, and temperature of the surface affect the characteristics of the FMF. Among recent papers, van der Ha formulate the FMF-induced force and torque effects on satellites in elliptical transfer orbit, ${ }^{7)}$ Hart et al. establish analytical coefficient models for typical surface shapes. ${ }^{8)}$ There is also a theoretical approach published by Sengers et al., 9) investigate the correlations of Knudsen number and speed ratio on the force coefficients.

In this paper, we focus on the FMF effects from the temperature variation of the satellite surfaces so that test cases are chosen to be 'easy-to-visualize' conditions. We employ a simple attitude pointing and single orbit configuration with seasonal variations in terms of epochs. These conditions allow us to obtain analytical expressions and results. Meanwhile, these analytical first-order estimates play an important role in the early phase of mission study and real-time operations as well. The current study intends to obtain those quick results while keeping the formulation as general as possible.

The aerodynamic effects are the dominant non-gravitational forces acting on an object below $600 \mathrm{~km}$ altitude orbit. The variation of the upper atmosphere is mainly driven by the heat input from the sun in the extreme ultraviolet wavelength, which causes the neutral density to change with several orders of magnitude. ${ }^{10)}$ Therefore, we employ the NRLMSISE-00 atmospheric model, ${ }^{11)}$ which is a statistical average of several measurements and suitable for our generic analysis.

Up to now, there are very limited investigations on the effects of surface temperature over the orbital motion, mainly due to the complexity of temperature calculations. Further refinements are foreseen by introducing the surface temperature estimation on the FMF calculations, and it would reveal the dynamic trend of the effect as well. And thus, we pursue a high fidelity calculation method of the FMF effects on a satellite by taking into account the orbit and attitude motion of the satellite.

\section{Geometry and Notations}

To perform a generic analysis on the FMF effects with temperature variations in orbit, the dynamics of the satellite are expressed in Earth centered inertial (ECI) frame. In addition, according to the emphasis on Earth observation satellites, the attitude pointing in this paper adopts a simple Earth pointing body with Sun tracking solar panels. The state (position, velocity, attitude) is calculated using the existing HPS modules with EGM2008 gravity field model with rotating atmosphere, ${ }^{12)}$ then the FMF and temperature variations are post processed with the given output from HPS.
Figure 2 shows the satellite-body fixed (BF) frame and notation angles which express the surface normals in $\mathrm{BF}$ frame. The attitude pointing is defined by the Sun and nadir directions as follows: The $X_{B F}$ and $Y_{B F}$ axes are aligned with the velocity vector and orbit normal, respectively, and the $Z_{B F}$ axis which points in the radial direction in ECI frame completes the coordinate. Thus, the $-\mathrm{Z}$ face of the satellite is kept pointing down to the Earth and this face is where mission payloads are expected to be mounted.

The planet surface and its radiation models are inherited from the established model, ${ }^{4,5)}$ while incorporating the Earth reflectivity data from NASA data archive. ${ }^{13)}$ The provided reflectivity data account for the average (or nominal) influences of clouds and surface conditions, e.g. ice and snow, from several satellite measurements.

The satellite model parameters are listed in Table 1. The body panels are covered by Multi-Layer Insulation (MLI) and the solar panels rotate around $\mathrm{Y}$ axis to keep them pointing towards the Sun (see Fig. 2). The surface properties of the satellite are provided with exemplary values, in the other hand, the surface temperature calculation includes the model of the emissivity of the solar panel back side. ${ }^{4,14)}$

Some straightforward and practical simplifications have been made to compute the view factor, which scales the radiative contributions of Earth albedo (ALB) and infrared radiation (IR) from the Earth's surface. ${ }^{4,5)}$ By using the calculated view factor, we obtain the energy inputs from ALB and IR on individual surface elements. These heat inputs together with incident solar flux are then used to compute the surface temperatures. One basic assumption on the incoming solar fluxes is that they are assumed to be parallel in LEO. This assumption can be employed without loosing generality and accuracy.

\section{Free-Molecular Flow}

The Knudsen number $K n$, which is the ratio between the mean free path of the molecules and a characteristic length of the object, characterizes the rarefied gas flow. Higher $K n$ corresponds to a flow in which the momentum exchanges are modeled by the molecular collisions on the object, so-called free-molecular flow. The variations in the atmosphere are very dynamic and thus difficult to model or measure in detail. However, empirically, the mean free path of the atmospheric molecules are in km-range above 200 [km] altitude.

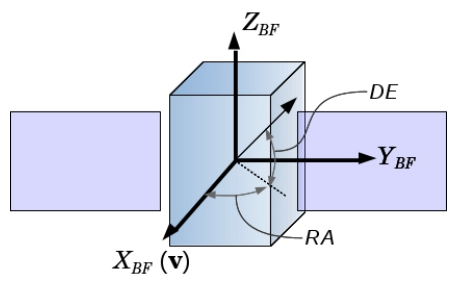

Fig. 2. Body frame and notation angles.

Table 1. Satellite model parameters.

\begin{tabular}{|l|l|}
\hline \multirow{2}{*}{ Dimensions [m] } & $1.5 \times 1.5 \times 2.0$ (body) \\
\cline { 2 - 2 } & $1.0 \times 1.5$ (solar panel) \\
\hline Emissivity (MLI) & 0.7 \\
\hline$\left[\alpha, \gamma_{\mathrm{d}}, \gamma_{\mathrm{s}}\right](\mathrm{MLI})$ & {$[0.72,0.24,0.04]$} \\
\hline
\end{tabular}




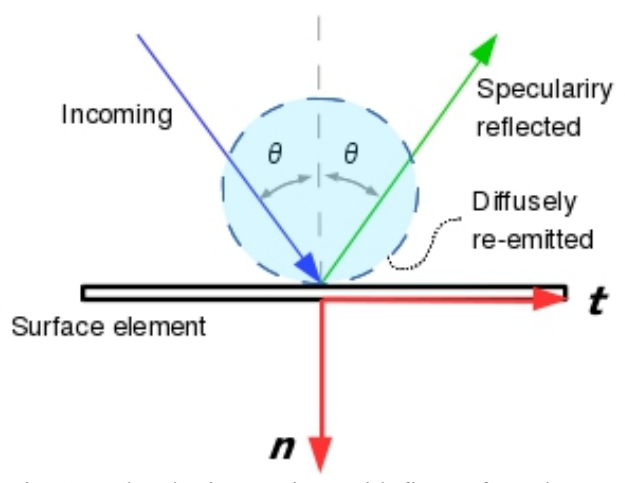

Fig. 3. Molecular interactions with flat surface element.

Figure 3 depicts the FMF surface interactions on a flat surface element. The representation of the aerodynamic force requires knowledge of the intricate interactions of the free-flow molecules with the surface exposed to the flow. These interactions are modeled with two independent phenomena, specular reflection and diffuse re-emission. Specular reflection assumes (ideal) elastic momentum exchange of a molecule with a surface and thus the molecule has the same magnitude of velocity with negative sign after the reflection and the resulting force is directed only along the negative surface normal (n, see Fig. 3).

Diffuse reflection has twofold processes and is assumed to be inelastic. Molecules, which are not specularly reflected, are first accommodated on the surface and this accommodation ratio is modeled with an energy accommodation coefficient. ${ }^{15)}$ These accommodated molecules are diffusely re-emitted from the surface and the most probable speed $v_{m}$ of re-emission can be expressed as a function of surface temperature

$$
v_{m}=\sqrt{\frac{2 k T_{w}}{m}}
$$

These particle-surface interactions are similar to the momentum exchange of a photon, well-known as SRP. In FMF, the surface temperature determines the speed of a molecule at the re-emission as shown in Eq. (1) and thus, the resulting recoil force.

We start with the FMF model presented by Klinkrad and Fritsche, ${ }^{16)}$ who express the aerodynamic force $\mathbf{F}$ exerted on a flat satellite surface $A$ in the form shown in Eq. (2) by using the local air density $\rho$. This $\rho$ represents the mean density of molecular species existing in the higher atmosphere, namely $\mathrm{N}_{2}, \mathrm{~N}, \mathrm{O}_{2}, \mathrm{O}, \mathrm{He}, \mathrm{H}$, and $\mathrm{Ar}$.

$$
\begin{aligned}
\mathbf{F} & =\frac{1}{2} \rho A v_{i}^{2} \mathbf{C} \quad[N] \\
& =\frac{1}{2} \rho A v_{i}^{2}\left\{\sigma_{d} \mathbf{C}_{d}+\sigma_{s} \mathbf{C}_{s}\right\} \\
\sigma_{s} & =1-\sigma_{d}
\end{aligned}
$$

The parameters of $v_{i}$ and $\mathbf{C}$ represent the magnitude of the incident free-stream velocity and the aerodynamic effect in vector components, respectively. The vector $\mathbf{C}$ contains the characteristics of the object, i.e. attitude, geometrical configuration, and surface properties. An important note is that the reflection coefficients $\left(\sigma_{s}\right.$ and $\left.\sigma_{d}\right)$ are different from the reflectivities $\left(\gamma_{s}\right.$ and $\left.\gamma_{d}\right)$ in SRP calculation.
When accounting for the directions of the resulting force components, we have : ${ }^{7)}$

$$
\begin{gathered}
\mathbf{C}_{d}=\left(\frac{1}{S}\right)^{2}\left[\left(\frac{\Pi\left(S_{n}\right)}{\sqrt{\pi}}+\sqrt{\frac{T_{w}}{T}} \frac{\chi\left(S_{n}\right)}{2}\right) \mathbf{n}+\left(\frac{S_{t} \chi\left(S_{n}\right)}{\sqrt{\pi}}\right) \mathbf{t}\right] \\
\mathbf{C}_{s}=\frac{2}{\sqrt{\pi}} \frac{\Pi\left(S_{n}\right)}{S^{2}} \mathbf{n}
\end{gathered}
$$

with

$$
\begin{gathered}
S=\frac{v_{i}}{v_{m}}, \quad S_{n}=S \cos \theta, \quad S_{t}=S \sin \theta \\
\Pi\left(S_{n}\right)=S_{n} \exp \left(-S_{n}^{2}\right)+\sqrt{\pi}\left(S_{n}^{2}+0.5\right)\left(1+\operatorname{erf}\left(S_{n}\right)\right) \\
\chi\left(S_{n}\right)=\exp \left(-S_{n}^{2}\right)+\sqrt{\pi} S_{n}\left(1+\operatorname{erf}\left(S_{n}\right)\right)
\end{gathered}
$$

Substituting these relations in Eq. (2), the components can be now expressed along negative surface normal $\mathbf{n}$ and its orthogonal direction $\mathbf{t}$,

$$
\begin{aligned}
\mathbf{F} & =\frac{1}{2} \rho A v_{i}^{2}\left\{C_{n} \mathbf{n}+C_{t} \mathbf{t}\right\} \\
& =\frac{1}{2} \rho A v_{i}^{2}\left\{\left(C_{n 1}+C_{n 2}\right) \mathbf{n}+C_{t} \mathbf{t}\right\}
\end{aligned}
$$

with

$$
\begin{aligned}
& C_{n 1}=\frac{2-\sigma_{d}}{\sqrt{\pi}} \frac{\Pi\left(S_{n}\right)}{S^{2}} \\
& C_{n 2}=\frac{\sigma_{d}}{2} \frac{\chi\left(S_{n}\right)}{S^{2}} \sqrt{\frac{T_{w}}{T}} \\
& C_{t}=\frac{\sigma_{d}}{\sqrt{\pi}} \frac{S_{t} \chi\left(S_{n}\right)}{S^{2}}
\end{aligned}
$$

These coefficients can be interpreted as, $C_{n l}$ represents the magnitude of the momentum exchange along $\mathbf{n}$ with local speed ratio $S, C_{n 2}$ accounts for the re-emission of the accommodated molecules with surface temperature along $\mathbf{n}$, and $C_{t}$ provides the shear force components due to the molecular accommodations along $\mathbf{t}$.

The coefficient $C_{n}\left(=C_{n 1}+C_{n 2}\right)$ is compatible with the conventional drag coefficient $C_{D} . C_{n}$ represents the force coefficient along the surface normal that is identical to drag coefficient when the surface is orthogonal to the flow. In the extreme of high speed ratio, it can be reduced to $C_{n} \approx 2\left(2-\sigma_{d}\right)$. Considering the range of $0.8 \leq \sigma_{d} \leq 1.0$ in practice, this amounts to $2.0 \leq C_{n} \leq 2.4$. In the following analysis, we employed $\sigma_{d}=0.9$.

The provided formulation of $C_{n 2}$ includes the surface temperature as a parameter, which changes dynamically in orbit. By employing the straightforward temperature calculation method, ${ }^{4,5}$ we could track its variation including the contributions from several radiation sources. The assumptions we made in temperature calculation are A). each surface element is thermally isolated from other elements, B). constant interior temperature, and $\mathrm{C}$ ). the resulting temperatures are obtained in steady state conditions.

Components in Eqs. (10)-(12) are computed in a discrete manner, including the estimated surface temperatures at each position and attitude. Since the surface temperature is one of the parameters of our interest, the temperature calculation incorporates ALB and IR as secondary heat sources to obtain realistic predictions of the effect. 


\section{Simulation}

In order to investigate the FMF effects with temperature variation, simple orbit and attitude conditions are employed, see Table 2 for the orbital elements. We use an exemplary orbit type of sun-synchronous orbit and 3 different epochs of summer/winter solstices and the vernal equinox in the year of 2000. Under each epoch, we compute satellite states, perturbations, surface temperatures, and resulting FMF effects over one orbital revolution.

The NRLMSISE-00 model is used to obtain the mean density and ambient temperature at a given spot in the orbit. Accordingly, we obtain the speed ratio, which is a ratio between the incident molecular velocity and the most probable speed of the molecules in the ambient atmosphere.

To investigate the seasonal variation, simulations are performed for 3 different seasons and Fig. 4 shows the speed ratio variation over one orbital period in each season. The speed ratios stay in the similar envelop throughout all seasons.

The attitude is fixed with velocity vector $\left(=X_{B F}\right)$ and nadir direction $\left(=-Z_{B F}\right)$ with Sun tracking solar panels. This represents a typical Earth observation satellite configuration. The orbit is also fixed with Local Time of Ascending Node (LTAN) of $0 \mathrm{pm}$ to include the eclipse where we expect significant temperature variations.

Table 2. Orbit condition.

\begin{tabular}{|c|c|c|c|c|}
\hline $\mathrm{a}[\mathrm{km}]$ & $\mathrm{e}$ & $\mathrm{i}[\mathrm{deg}]$ & $\omega[\mathrm{deg}]$ & LTAN \\
\hline 6878.1 & 0.001 & 97.401 & 0 & 0 pm (noon) \\
\hline
\end{tabular}

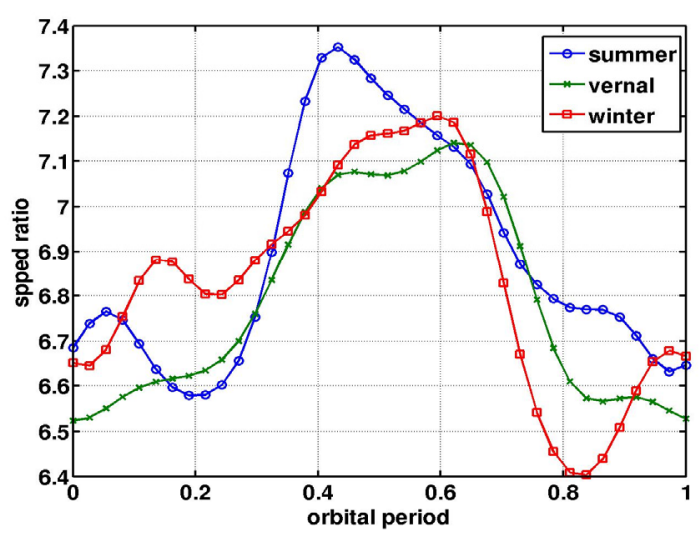

Fig. 4. Speed ratio in $500[\mathrm{~km}]$ orbit at solstices and equinox.

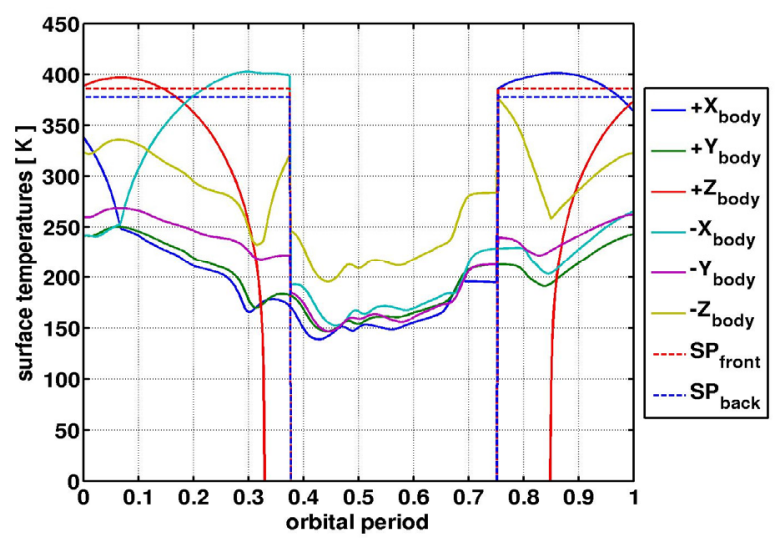

Fig. 5. Surface temperatures over one revolution.
These conditions lead to the results that mainly 5 surface elements are exposed to the flow. Namely, they are $+\mathrm{X}$ body, 2 solar panel front / back surfaces. There are small non-zero components of the FMF effect seen also on the other body surfaces due to the orbit inclination and eccentricity. However, in this paper, we focus on the major components acting on the $+\mathrm{X}$ body and the solar panel front side.

The obtained surface temperatures at summer epoch are shown in Fig. 5. Since the temperature calculation is based on steady state condition with straightforward heat balance, the temperature drops to $0[\mathrm{~K}]$ if a surface receives no energy input. In addition, we employed a cylindrical eclipse model for flux calculations based on parallel solar flux assumption.

\section{Result and Discussion}

For the initial illustration of the temperature contribution, we first look into the $+\mathrm{X}$ body surface, which is kept normal to the velocity vector and thus represents a flat-plate configuration normal to the flow. The obtained magnitude of the FMF force and coefficients in summer epoch are shown in Figs. 6 and 7.

Simple atmospheric drag results with drag coefficients of $C_{D}=2.0,2.5$ are included in Fig. 6 for comparison, and Fig. 7 shows the distribution of $C_{n}$ components from Eqs. (10) and (11). Here, the simple atmospheric drag components are calculated by substituting specified drag coefficients in $\mathbf{C}$, in the first line of Eq. (2) with same area and velocity.

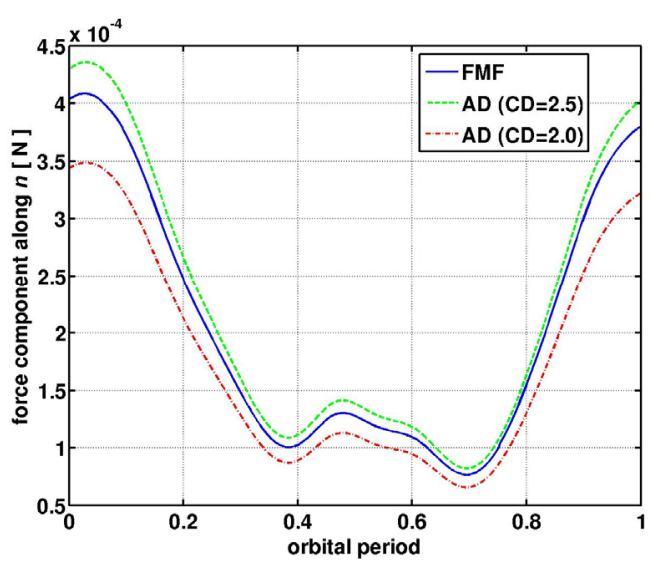

Fig. 7. Magnitude of the FMF force component on $+\mathrm{X}$ body

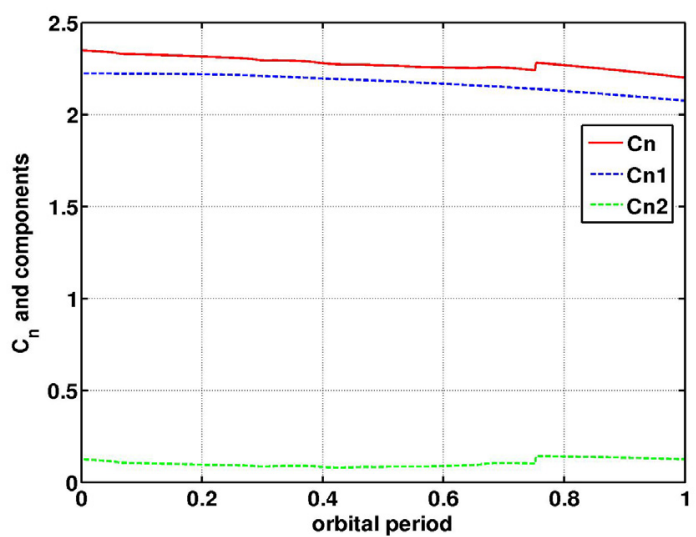

Fig. 6. FMF force coefficient and components of $+\mathrm{X}$ body (summer). 


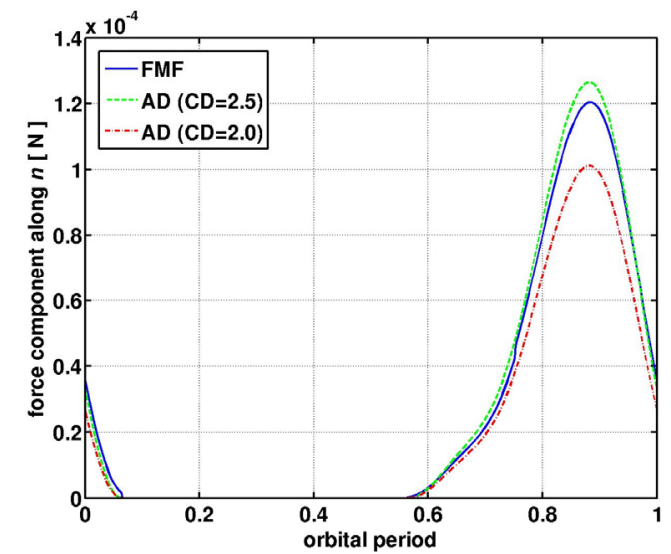

Fig. 8. Magnitude of the FMF force component on solar panel front, along $\mathbf{n}$ (summer).

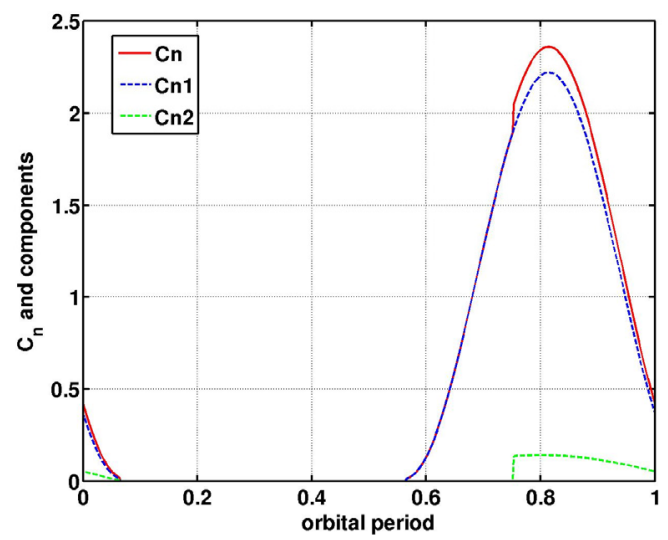

Fig. 9. FMF force coefficient and components $C_{n}$ of solar panel front, along $\mathbf{n}$ (summer).

The FMF results basically stay within the two boundaries of simple atmospheric drag. However, we find a jump in $C_{n 2}$ component in Fig. 7. The jump corresponds to the exit of the eclipse condition as expected and has about 6.3 [\%] contribution on total $C_{n}$. The same discussion applies to the Sun-tracking solar panel, shown in Figs. 8 and 9.

The $C_{n 2}$ jump is more clearly seen in Fig. 9, where $C_{n 1}$ corresponds to the angle between the surface normal and the velocity vector, while $C_{n 2}$ materializes only under the illuminated condition (see also, Fig. 5). The magnitude of the jump due to the $C_{n 2}$ on the solar panel front is, similar to $+\mathrm{X}$ body, about $6.6[\%]$ with respect to the total $C_{n}$.

The results of Figs. 6 - 9 are summarized in Table 3 for the ranges of each component. The magnitude of the FMF force and $C_{n}$ vary due to the dynamical change of the atmosphere over the orbital motion. The primal factor is the diurnal variations of atmospheric molecular concentration, where heavier molecules stay near the subsolar point and lighter molecules gather around the anti-subsolar point. ${ }^{16)}$

Table 3. $C_{n}$ and FMF force variations over one orbit in summer.

\begin{tabular}{|l|c|c|c|c|}
\hline \multirow{2}{*}{} & \multicolumn{2}{|c|}{$C_{n}$} & \multicolumn{2}{c|}{ FMF force along $\mathbf{n}[\mathrm{N}]$} \\
\cline { 2 - 5 } & $\max$ & $\min$ & $\max$ & $\min$ \\
\hline$+\mathrm{X}$ body & 2.35 & 2.20 & $4.09 \times 10^{-4}$ & $7.66 \times 10^{-5}$ \\
\hline Solar panel & 2.36 & 0 & $1.20 \times 10^{-4}$ & 0 \\
\hline
\end{tabular}

The observed jump in $C_{n}$ might be exaggerated with first-order estimation of the analytical temperature calculations, based on the steady state conditions. However, it generates the disturbance on the attitude motion and might explain many of the reported spikes on orbit. ${ }^{17)}$ Further detailed analysis including attitude effects are in progress.

Additionally, in the case of pursuing higher precision in application, we need more sophisticated and dedicated numerical analysis including the temperature variation with surface properties.

Next, we spot on the FMF effects on a rotating surface, in our case the solar panel. The proportional distributions of $C_{n}$ on a solar panel front for each epoch are shown in Figs. 10 12 The contributions of respective components are shown in the stacked-bar and given in [\%].

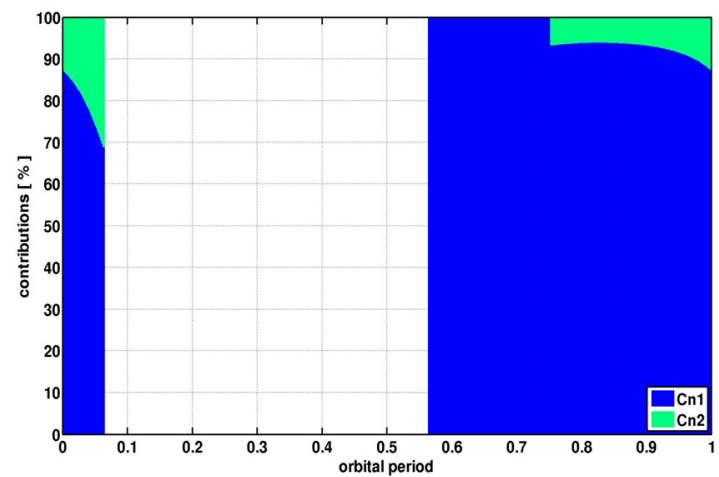

Fig. 10. $C_{n 1}$ and $C_{n 2}$ contributions on solar panel front (summer).

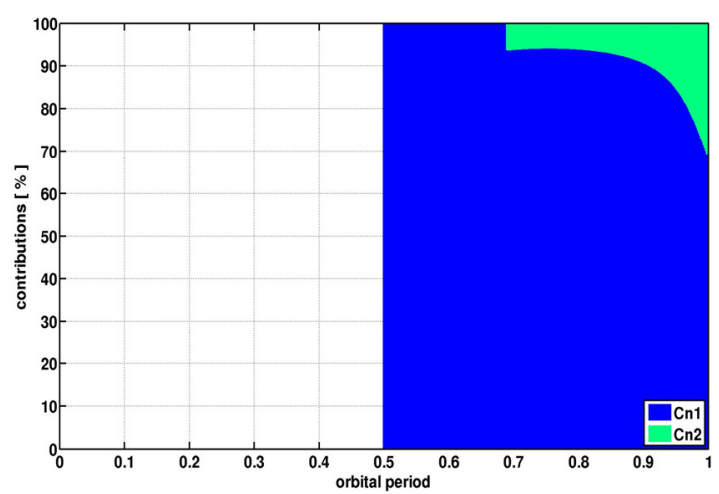

Fig. 11. $C_{n 1}$ and $C_{n 2}$ contributions on solar panel front (vernal).

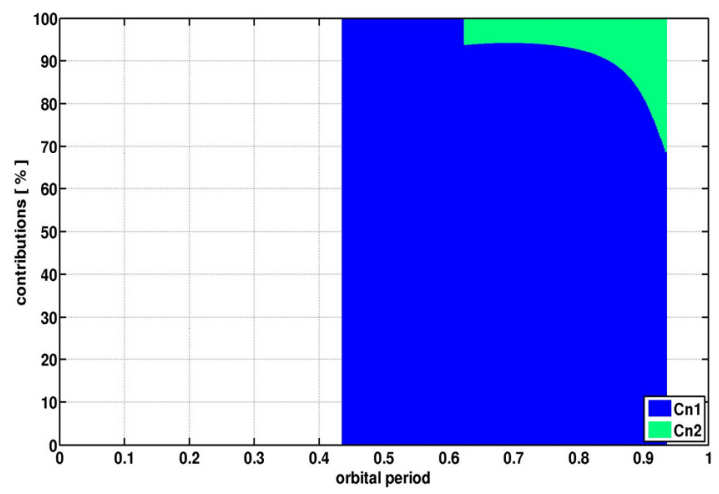

Fig. 12. $C_{n 1}$ and $C_{n 2}$ contributions on solar panel front (winter). 
Table 4. Characteristic results of temperature contributions on solar panel front with different epochs (Figs. $10-12$ ).

\begin{tabular}{|c|c|c|c|c|}
\hline \multirow{2}{*}{} & \multirow{2}{*}{$C_{n}(\max )$} & \multicolumn{3}{|c|}{$C_{n 2}$} \\
\cline { 3 - 5 } & & Max. & Jump & Jump in [\%] \\
\hline Summer & 2.36 & 0.141 & 0.135 & $6.61 *$ \\
\hline Vernal & 2.36 & 0.136 & 0.128 & $6.23 *$ \\
\hline Winter & 2.35 & 0.132 & 0.125 & $6.10^{*}$ \\
\hline Deflection & 0.01 & 0.009 & 0.01 & 0.51 \\
\hline
\end{tabular}

The gaps seen in the Figs. $10-12$ are the orbital arcs where the surface is not exposed to the atmospheric flow, and are not from the eclipse condition. Due to the initial true anomaly of 0 with different epoch, these conditions shift within the orbital period and have slightly different peak values of $C_{n}$ components.

Table 4 summarizes the obtained characteristics of temperature contributions from Figs. 10 - 12. The listed values of $C_{n 2}$ are slightly decreasing from summer to winter, but the seasonal deflection of total $C_{n}$ is negligible at only 0.4 [\%] of the maximum value. In addition, the ratios of the jump in $C_{n 2}$ are above $6[\%]$ throughout the seasons. Thus, no remarkable seasonal variations are found under the selected conditions.

In Figs. 10 - 12, contributions of $C_{n 2}$ have peaks about 30 [\%] of total $C_{n}$. This is the combined effect of small $S_{n}$ and surface temperature. The $C_{n l}$ component rapidly approaches 0 with the large separation angle between surface normal and velocity vector. On the other hand, $C_{n 2}$ remains due to the almost constant surface temperature. Although, the value of $C_{n}$ at the spot is less than $1[\%]$ of the maximum of $C_{n}$ on solar panels, the effects are practically negligible (see Figs. 8 - 10).

In all cases, the maximum value of $C_{n 2}$ takes place slightly after the exit of eclipse. These shifts are induced by the ALB and the IR radiations from visible surfaces of Earth that are illuminated by the Sun. Thus, the trend and its magnitude vary with specific mission parameters on the orbital geometry, satellite properties, and attitude.

\section{Conclusion}

The dynamic variations of the free-molecular flow effects are shown with the new aspect of temperature contributions based on analytical formulations. The chosen exemplary test cases indicate that the magnitude of the force coefficient change is around $6 \%$, which is induced by the surface temperature variation at the exit of eclipse.

On the other hand, the seasonal variations on the free-molecular flow effects are found to be small. However, the incorporation of the surface temperature leads to identify dynamical variations and spikes around the eclipse boundary. This is of specific interest in the scientific data extraction of gravimetry missions. The research continues in the attitude effects and more precise orbit propagation methodology.

Since the atmosphere is very hard to model precisely, the results shown here do not guarantee the generality of the discussion. Nevertheless, the temperature contribution on the free-molecular flow calculation is now foreseen to be implemented in our mission analysis software HPS-SG for the sake of capturing dynamic characteristics on orbit and attitude.

\section{Acknowledgments}

This research is supported by the Sonderforschungsbereich (SFB) grant from Deutsche Forschungsgemeinschaft (DFG), "Relativistic geodesy and gravimetry with quantum sensors-modeling, geo-metrology, and future technologygeo- $Q$ " (SFB 1128). Additionally, the authors would like to acknowledge supports by the German Space Agency (DLR) with funds of the BMWi (FKZ 50 OY 1305) and the DFG (LA 905/12-1).

\section{References}

1) Reigber, C., Schwintzer, P., Lühr, H. : The CHAMP geopotential mission, Bolletitino Di Geofisica Teorica df Applicata, 40 (1999), pp. 285-289.

2) Tapley, B. D., Bettadpur, S., Watkins, M., Reigber, C. : The Gravity Recovery and Climate Experiment : Mission Overview and Early Results, Geophysical Research Letters, 31(2004), doi : 10.1029/2004GL019920.

3) Drinkwater, M. R., Haagmans, R., Muzi, D., Popescu, A., Floberghagen, R., Kern, M., Fehringer, M. : The GOCE Gravity Mission : ESA's First Core Earth Explorer, Proceedings of 3rd International GOCE User Workshop, 2007, pp. 1-8.

4) Kato, T., Rievers, B., van der Ha, J., Lämmerzahl, C. : Sensitivity Analysis of the Non-Gravitational Perturbations on a Mercury Orbiter, Advances in the Astronautical Sciences, 143 (2012), pp. 1579-1595.

5) Kato, T., Theil, S. van der Ha, J. : External Torques Affecting the Attidue Motion of a Mercury Orbiter, Advances in the Astronautical Sciences, 152 (2014), pp.3475-3493.

6) Pelivan, I., Heidecker, A., Theil. S. : High Performance Satellite Dynamics and Control Simulation for Multi-Purpose Application, Journal of Aerospace Engineering, Sciences and Applications, 4 (2012), pp.119-130.

7) van der Ha, J. : Free-Molecular Flow Induced Attitude Changes of a Spinning Satellites in Eliptical Orbits, Advances in the Astronautical Sciences, 148 (2013), pp. 1321-1340.

8) Hart, K., Dutta, S., Simonis, K., Steinfeldt, B., Braun, R. Analytically-derived Aerodynamic Force and Moment Coefficients of Resident Space Objects in Free-Molecular Flow, Proceedings of AIAA Atmospheric Flight Mechanics Conference, January, 2014, AIAA 2014-0728, doi: 10.2514/6.2014-0728

9) Sengers, J. V., Lin Wang, Y.-Y., Kamgar-Parsi, B., Dorfman, J. R. : Kinetic Theory of Drag on Objects in Nearly Free Molecular Flow, Physica A : Statistical Mechanics and its Applications, 413(2014), pp.409-425, doi : 10.1016/j.physa.2014.06.026.

10) Storz, M. S., Bowman, B. R., Major Branson, J. I., Casali, S. J., Tobiska, W. K. : High Accuracy Satellite Drag Model (HASDM), Advances in Space Research, 36 (2005), pp.2497-2505.

11) Picone, J. M., Hedin, A. E.,Drob, D. P. : NRLMSISE-00 Empirical Model of the Atmosphere : Statistical Comparisons and Scientific Issues, Journal of Geophysical Research, 107(2002), No.A12, 1486, doi : 10.1029/2002JA009430.

12) Förste, C., Schmidt, R., Stubenvol, R., Flehtner, F., Meyer, U., König, R., et al.: The GeoForschungsZentrum Potsdam/Groupe de Recherche de Geodesie Spatiale Satellite-Only and Combined Gravity Field Models: EIGEN-GL04S1 and EIGEN-GL04C, Journal of Geodesy, 82(2008), pp 331-346.

13) NASA Goddard Space Flight Center, data archive ftp://toms.gsfc.nasa.gov/pub, (cited on April 21st, 2015).

14) Kato, T., van der Ha, J.: Precise Modelling of Solar and Thermal Accelerations on Rosetta, Acta Astronautica, 72(2012),pp 165-177.

15) Schaaf, S. A., Chmbre, P. L.: Flow of Rarefied Gases, Princeton University Press, 1961.

16) Klinkrad, H., Fritsche, B.: Orbit and Attitude Perturbations due to Aerodynamics and Radiation Pressure, ESA Workshop on Space Weather, ESTEC, Noordwijk, the Netherlands, 11-13 November 1998.

17) Flury, J., Bettadpur, S., Tapley, B. D. : Precise Accelerometry onboard the GRACE gravity Field Satellite Mission, Advances in Space Reserach, 42(2008), pp. 1414-1423. 